\title{
Steel's recyclability: demonstrating the benefits of recycling steel to achieve a circular economy
}

\author{
Clare Broadbent ${ }^{1}$
}

Received: 25 May 2015 / Accepted: 25 February 2016 /Published online: 21 March 2016

(C) The Author(s) 2016. This article is published with open access at Springerlink.com

\begin{abstract}
Purpose In a world where the population is expected to peak at around 9 billion people in the next 30 to 40 years, carefully managing our finite natural resources is becoming critical. We must abandon the outdated 'take, make, consume and dispose' mentality and move toward a circular economy model for optimal resource efficiency. Products must be designed for reuse and remanufacturing, which would reduce significant costs in terms of energy and natural resources.

Methods To measure progress in achieving a circular economy, we need a life cycle approach that measures the social, economic and environmental impact of a product throughout its full life cycle - from raw material extraction to end-of-life (EoL) recycling or disposal. Life cycle thinking must become a key requirement for all manufacturing decisions, ensuring that the most appropriate material is chosen for the specific application, considering all aspects of a products' life. The steel industry has been developing LCI data for 20 years. This is used to assess a product's environmental performance from steel production to steel recycling at end-of-life. The steel industry has developed a methodology to show the benefits of using recycled steel to make new products. Using recycled materials also carries an embodied burden that should be considered when undertaking a full LCA.

Results and discussion The recycling methodology is in accordance with ISO 14040/44:2006 and considers the environmental burden of using steel scrap and the benefit of scrap
\end{abstract}

Responsible editor: Andrea J. Russell-Vaccari

Clare Broadbent

broadbent@worldsteel.org

1 World Steel Association, 120 rue Colonel Bourg, 1140 Brussels, Belgium recycling from end-of-life products. It considers the recycling of scrap into new steel as closed material loop recycling, and thus, recycling steel scrap avoids the production of primary steel. The methodology developed shows that for every $1 \mathrm{~kg}$ of steel scrap that is recycled at the end of the products life, a saving of $1.5 \mathrm{~kg} \mathrm{CO}_{2}$-e emissions, $13.4 \mathrm{MJ}$ primary energy and $1.4 \mathrm{~kg}$ iron ore can be achieved. This equates to 73,64 and $90 \%$, respectively, when compared to $100 \%$ primary production.

Conclusions Incorporating this recycling methodology into a full LCA demonstrates how the steel industry is an integral part of the circular economy model which promotes zero waste; a reduction in the amount of materials used and encourages the reuse and recycling of materials.

Keywords Circular economy $\cdot$ Cradle to grave $\cdot$ LCA . Recycling $\cdot$ Steel

\section{Introduction}

\subsection{The circular economy}

Steel is everywhere in our lives and is at the heart of a sustainable future. The steel industry is an integral part of the global circular economy. The circular economy is a move from linear business models, in which products are manufactured from raw materials and then discarded at the end of their useful lives, to circular business models where intelligent design leads to products or their parts being repaired, reused, returned and recycled (World Economic Forum 2014). A circular economy aims to rebuild capital, whether it is financial, manufacturing, human, social or natural. This approach enhances the flow of goods and services (Ellen MacArthur Foundation 2014). The concept of the 
circular economy drives optimal resource efficiency. It makes sure that resources are efficiently allocated to products and services in such a way as to maximise the economic wellbeing of everyone. In addition, products need to be designed to be durable, easy to repair and, ultimately, to be recycled. The cost of reusing, repairing or remanufacturing products has to be competitive to encourage these practices. Simply replacing a product with a new one should no longer be the norm.

A circular economy ensures that value is maintained within a product when it reaches the end of its useful life while at the same time reducing or eliminating waste. This idea is fundamental to the triple-bottom-line concept of sustainability, which focuses on the interplay between environmental, social and economic factors. In a well-structured circular economy, the steel industry has significant competitive advantages over competing materials and these can be demonstrated through a life cycle approach.

\subsection{Life cycle assessment in the steel industry}

The World Steel Association (worldsteel) has been developing a database of life cycle inventories (LCI) of steel products for more than 20 years together with an externally reviewed methodology report. This LCI database of 15 steel products accounts for the cradle to gate steel production, including raw material mining and manufacturing, as well as accounting for the benefits of recycling steel from products at the end of their life. This database and methodology assist LCA practitioners modelling steel products to carry out full cradle to grave life cycle assessments. This report demonstrates what approaches are currently available for including recycling in LCA and the rationale for the approach that the steel industry has decided to use based on the closed material loop recycling methodology. A detailed account of the methodology is provided, which demonstrates the environmental value of recycling steel from products when they reach the end of their useful life.

\section{Current practice for recycling methodologies}

\subsection{Existing recycling methodologies}

The three main approaches to recycling which form the basis for many discussions are the following:

\section{- Cut-off approach (100-0)}

The cut-off approach considers the impacts and/or benefits of recycling that only occur within the product system being studied. There is no crediting or assignment of environmental impacts between different product systems, and metal scrap at the point of discard is considered to have no upstream environmental impacts beyond remelting. This is also known as the recycled content method because the benefits of metals recycling are only taken into account on the input side (considered as being 'free') and recycling at end-of-life is neglected regardless of recycling rate. From a policy perspective, this method leads to a focus on increasing the percentage of recycled materials in the product. Figure 1 shows how the cut-off approach would be applied throughout the life cycle.

- End-of-life approach (0-100)

The end-of-life approach takes an overall approach to recycling as it considers the assignment of environmental impacts and credits between different product systems across different life cycles and the environmental impact of the product system is dependent on the recycling rate at end-of-life. Where a material is recycled at end-of-life, the product system is credited with an avoided burden based on the reduced requirement for virgin material production in the next life cycle. Equally, any recycled content adds the same burden to the product system, per kilogram of steel scrap, in order to share the burden with the previous life cycle. This method is also known as the closed material loop method because recycling saves the production of virgin material with the same properties. From a policy perspective, this method encourages the recycling of products at the end of their life. Figure 2 shows how this approach would be applied for each stage of the life cycle; the impacts from the disposal of steel, if any, are negligible. Note that the amount of scrap used in the production of steel is typically lower than the amount of scrap recycled at end-of-life in the primary production route or for the secondary production route in the cases where a large amount of direct reduced iron or hot metal is used.

- The 50:50 method

This method falls half way in between the cut-off approach and end-of-life approach. For this reason, it

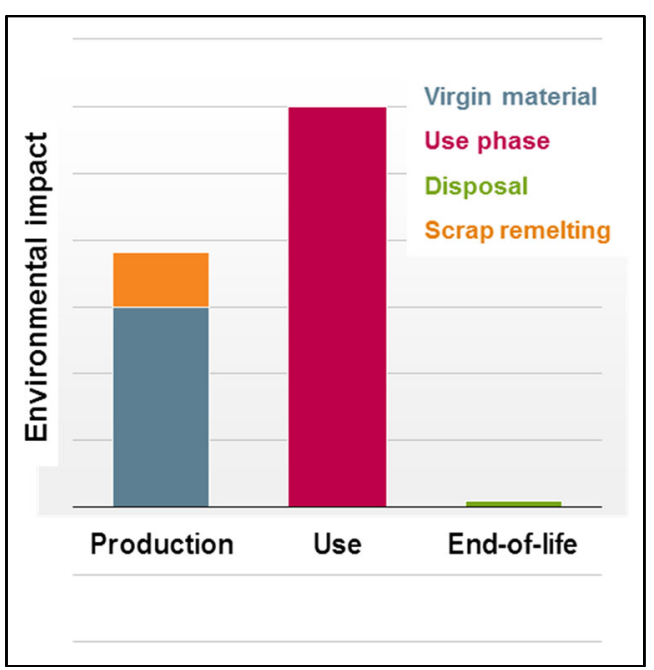

Fig. 1 Cut-off approach for a product system that uses virgin metal and recycled metal inputs 


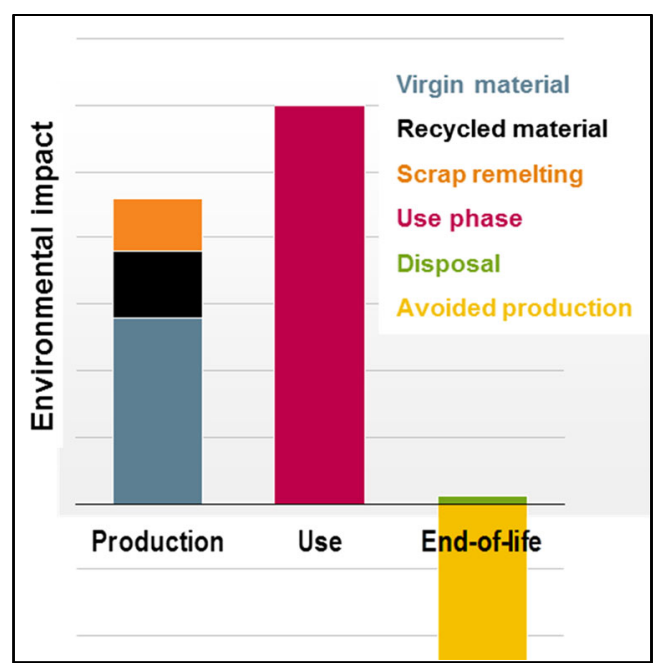

Fig. 2 End-of-life approach for a product system that uses both primary and recycled steel inputs

is seen as a compromise method, which credits both recycled content and end-of-life recycling. This method, although a compromise, can be a solution for systems where it is not clear if it is beneficial to provide incentives for recycled content or recycling at end-of-life.

In addition, there are multiple frameworks that address the incorporation of the benefits of recycling at the end of a product's life. Some examples of such publications are the following:

- World Resources Institute/World Business Council for Sustainable Development standards developed under the GHG Protocol Initiative (The Greenhouse Gas Protocol 2004)

- PAS 2050: Publicly Available Specification 2050: Specification for the assessment of the life cycle greenhouse gas emissions of goods and services (British Standards Institute 2008)

- EN 15804: Sustainability of construction works (European Committee for Standardisation 2013)

- ISO TS 14067: Carbon footprint of products (ISO TS 14067 2013)

- ILCD: The European Commission's International Reference Life Cycle Data System Handbook (European Commission 2010)

- The European Commission's Product Environmental Footprint (European Commission 2013)

The Declaration by the Metals Industry on Recycling Principles (Atherton et al. 2007) clearly defines the distinction between the recycled content approach and the end-of-life approach and why the latter is supported by the metals industry. The end-of-life approach encourages the recycling of products at the end of their life and therefore reduces waste going to landfill and saves the use of natural resources in creating new products - these are both key to a circular economy.

The European Commission's Product Environmental Footprint standard is currently in the pilot phase, and one of the aspects of this phase is to assess the methodology that has been defined for the end-of-life of products. This is being addressed by the different pilot projects including the metals industries.

\subsection{Steel recycling practice}

In the manufacture of steel, the term 'primary production' generally refers to the manufacture of iron (hot metal) from iron ore in a blast furnace $(\mathrm{BF})$, which is subsequently processed in the basic oxygen furnace (BOF) to make steel. 'Secondary production' refers to the 'recycling' route and is typically the electric arc furnace (EAF) process, which converts scrap into new steel by re-melting old steel. However, primary steel production is not unique to the BOF route, and similarly, secondary steel production is not unique to the EAF. It is common practice to use 10-30\% scrap as iron input in the BOF route. Primary steel production also occurs in the EAF route, when pre-reduced iron is used as a feedstock to the EAF process. This is demonstrated in Fig. 3.

Steel is $100 \%$ recyclable and scrap is converted to the same (or higher or lower) grade steel depending upon the metallurgy and processing of the required product. Some recycled products such as rebar require minimal processing, whilst the higher value engineering steels require more metallurgical and process controls to meet tighter specifications. The final economic value of the product is not determined by recycled content, and there are many examples of high value products that contain large amounts of recycled steel. Some steel products are principally sourced via the primary route mainly because the steel specifications require low residual elements and this can be achieved most cost-effectively using more primary material. In most cases, scrap with a low amount of residual elements commands a higher market price owing to the ease of processing through the recycling routes.

The growing global demand for steel results in a continuing capacity to absorb steel scrap. There is not enough scrap arising to manufacture all the steel required to satisfy the market. This is not a consequence of deficiencies in collecting scrap as the recovery rates of steel products are high and the lifetime of products is often long. Moving towards a circular economy, if more scrap becomes available, this could result in an increase in the proportion of steel made in the EAF route. Continuing improvements in the scrap processing plants and segregation of scrap types will improve efficiencies in the steelmaking process. 
Fig. 3 Connection between primary and secondary steel production

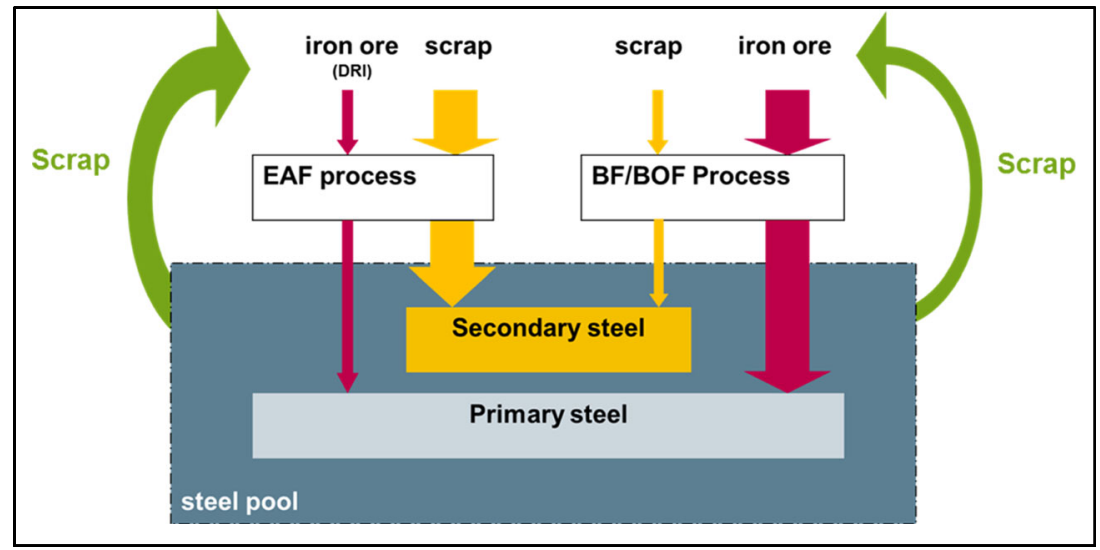

\section{Worldsteel's rationale for the chosen recycling approach}

The worldsteel LCI data collection methodology covers steel production from cradle to gate and in addition takes account of recycling of steel scrap in the following ways:

- Allocation for scrap inputs to the steelmaking process

- Allocation for steel scrap outputs from whole product system (e.g. scrap arising from an end-of-life building or vehicle)

Where systems have both scrap inputs and outputs, it is necessary to apply consistent allocation procedures to both, as is described in the worldsteel method.

This methodology is reviewed to conform to ISO 14044: 2006, which sets out allocation procedures for reuse and recycling. Within this standard, a distinction is made between open and closed loop recycling. Open loop recycling is used to describe product systems where material is recycled into a new different product or where inherent material properties change. Closed loop recycling applies to products that are recycled to produce the same product type or where the inherent material properties do not change. Where inherent material properties do not change, this is also known as closed material loop recycling.

The vast majority of steel recycling involves re-melting scrap to produce new steels with no change in the inherent properties of the basic steel material, and therefore, steel recycling can be regarded as closed loop. In this situation, ISO 14044:2006 states that 'in such cases, the need for allocation is avoided since the use of secondary material displaces the use of virgin (primary) materials'. This guidance provides the basis for the 'closed material loop' recycling methodology employed by worldsteel, which is used to deal with scrap inputs and outputs, and is recommended to be used for all LCA studies containing steel.
The choice of recycling methodology can depend on not only the goal and scope of the study but also the recycling system for the material used in the product life cycle. In the worldsteel methodology, the rationale for applying the closed material loop method as default is that

1. Steel scrap has significant economic value, so scrap is recovered, and it will be used for recycling. There is no need to create a demand for recycled material as this is already well established.

2. Steel is recycled in a closed material loop; the inherent properties of the primary and secondary product are equivalent, and thus, secondary material displaces primary production.

3. The magnitude of steel recycling is driven by end-of-life recycling rates and an end-of-life approach captures the impact of different recycling rates, regions and endproduct categories.

4. The demand for steel scrap exceeds the availability of the scrap. This is magnified partly due to the long lifetime of steel products. Designing products for easier end-of-life disassembly and recycling will enable more steel scrap to be recycled.

Using the closed material loop methodology, recovered steel scrap for recycling is usually allocated a credit (or benefit). When scrap is used in the manufacture of a new product, there is an allocation (or debit) associated with the scrap input. In this way, the benefit of net scrap arising or the debit of net scrap input can be accounted. Based on guidance from ISO 14044:2006, this scrap is allocated a value associated with avoided impacts such as an alternative source of equivalent (virgin) ferrous metal.

In the case of steel, the best approximation for the virgin product replaced by using scrap is the first recognisable steel product, which is cast steel or steel slab. Secondary steel from scrap (in the EAF route) avoids primary steel from the BOF route. With this approach, the allocation for scrap needs to be 
adjusted to take account of the scrap/steel yield associated with secondary steel making.

The worldsteel methodology follows the end-of-life approach because it accounts for the full life cycle of a product, from cradle to grave, the grave being the furnace into which the steel scrap is recycled.

\section{Worldsteel methodology for scrap recycling}

The worldsteel methodology for the use of steel scrap in the steelmaking process and the production of steel scrap at the end-of-life of a product is described in detail in the following sections.

\subsection{Terminology required}

A number of parameters relating to steel and recycling which will be used in the following equations are as follows:

1. Recovery rate $(R R)$ : the fraction of steel recovered as scrap during the lifetime of a steel product, including scrap generated after manufacturing the steel product under analysis. A value of $85 \%$ has been used in Eq. 11 .

2. Metallic yield $(Y)$ : the process yield (or efficiency) of the EAF. It is the ratio of steel output to scrap input (i.e. $>1-\mathrm{kg}$ scrap is required to produce $1-\mathrm{kg}$ steel). This is calculated using Eq. 4 as 1.092 based on worldsteel data published in 2010.

3. LCI for BOF steel production $\left(X_{B O F}\right)$ : the LCI for steel production from the BOF, which includes scrap. The value used in Eq. 8 of $1.756 \mathrm{~kg} \mathrm{CO}_{2}$ is from the worldsteel data published in 2010.

4. LCI for primary steel production $\left(X_{p r}\right)$ : the theoretical LCI for $100 \%$ primary metal production, from the BOF route, assuming $0 \%$ scrap input.

5. LCI for secondary steel production $\left(X_{r e}\right)$ : the LCI for $100 \%$ secondary metal production from scrap in the $\mathrm{EAF}$, assuming scrap $=100 \%$. The value used in Eq. 8 of $0.386 \mathrm{~kg} \mathrm{CO}_{2}$ is from the worldsteel data published in 2010.

6. The letter $\mathrm{X}$ in each of these terms refers to any LCI parameter, e.g. natural gas, $\mathrm{CO}_{2}$, water and limestone.

7. $S$ is the amount of scrap used in the steelmaking process to make a specific product. The value of $0.121 \mathrm{~kg}$ used in Eq. 11 for hot rolled coil is from the worldsteel data published in 2010.

\subsection{The LCI of steel scrap}

The methodology assumes the burdens of scrap input and the credits for recycling the steel at the end of the life of a product are equal, per kilogram, and that all scrap is treated equally. In reality, there are numerous grades of steel products, and therefore, steel scrap grades and a combination of these scrap types are used when making steel. It has not been feasible to calculate an LCI for each scrap grade, but this could be addressed in the future. As the use of scrap replaces the production of crude steel, and not a finished steel product, it is appropriate to assume a generic scrap grade for the purpose of these calculations. For coated or galvanised scrap grades, this will result in an overestimation of the burden for the scrap input (the yield will be lower) so will give more conservative results.

Collecting scrap at the end of the product's life and recycling it through the steel making process enables the saving of primary, virgin steel production.

This is commonly referred to as the integrated or BOF steel making route, but in reality, some steel scrap is always required in the process as it acts as a coolant in order to maintain the thermal balance in the process. Thus, there is no process using $100 \%$ virgin material (with $0 \%$ scrap input), and this theoretical value therefore needs to be calculated (see Sect. 6.3).

Furthermore, it is not the scrap itself that replaces this primary steel, as the scrap needs to be processed or recycled to make new steel. The EAF process is an example of $100 \%$ scrap recycling, though some EAFs also use hot metal or direct reduced iron (DRI) as an input to the process.

Finally, the EAF process is not $100 \%$ efficient, i.e. it needs more than $1 \mathrm{~kg}$ of scrap to make $1-\mathrm{kg}$ steel.

The LCI associated with the scrap, ScrapLCI, is thus equal to the credit associated with the avoided primary production of steel (assuming $0 \%$ scrap input), minus the burden associated with the recycling of steel scrap to make new steel, multiplied by the yield of this process (see Fig. 4) to consider losses in the process (see Sect. 4.1 for definitions):

ScrapLCI $=\left(X_{p r}-X_{r e}\right) Y$

The letter $X$ in each of these terms refers to any LCI parameter, e.g. natural gas, $\mathrm{CO}_{2}$ and water. The $\mathrm{CO}_{2}$ for scrap would be calculated as follows:

$\mathrm{CO}_{2} \mathrm{Scrap}=\left(\mathrm{CO}_{2 p r}-\mathrm{CO}_{2 r e}\right) \mathrm{Y}$

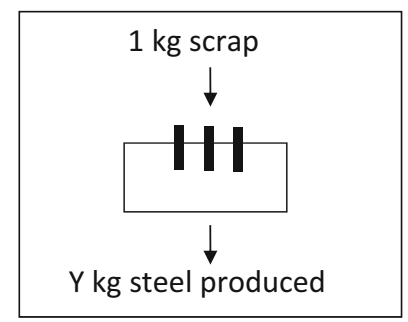

Fig. 4 The yield of the EAF process 
$Y$ is the process yield of the EAF (i.e. $>1-\mathrm{kg}$ scrap is required to produce $1-\mathrm{kg}$ steel)

The values for $X_{r e}$ and $Y$ are known by the industry as these values come from the steel producers. However, the theoretical value of $X_{p r}$ needs to be calculated.

\subsection{Theoretical value of $100 \%$ primary BOF steel, $X_{p r}$}

The theoretical value of $100 \%$ primary steel is calculated based on the LCI of steel slab made by the primary, or BOF route. As the steel slab contains a certain amount of scrap, this needs to be 'removed' from the LCI so that only virgin steel is accounted for, see Fig. 5a.

The scrap input to the BOF process (m kg scrap per 1-kg steel produced) that needs to be removed would be melted in the EAF process producing $\mathrm{mY} \mathrm{kg}$ steel, $Y$ being the yield of the steelmaking process. Therefore, the theoretical $100 \%$ primary route, $X_{p r}$, needs to produce 1-mY kg steel, see Fig. $5 \mathrm{~b}$.

In effect,

$X_{B O F}=(1-m Y)\left(X_{p r}\right)+m Y X_{r e}$

where $m$ is the scrap input to the BOF route $\left(\operatorname{Scrap}_{\mathrm{BOF}}\right)$ and $Y$ is the inverse of the scrap input to the EAF, Scrap re, i.e.

$Y=\frac{1}{\text { Scrap }_{\text {re }}}$

Therefore,

$m Y=\frac{\operatorname{Scrap}_{B O F}}{\operatorname{Scrap}_{r e}}$
This would then give the following:

$X_{\text {BOF }}=\left(1-\frac{\text { Scrap }_{B O F}}{\text { Scrap }_{r e}}\right)\left(X_{p r}\right)+\left(\frac{\text { Scrap }_{B O F}}{\text { Scrap }_{r e}}\right) X_{r e}$

Rearranging this equation will enable the theoretical value for $100 \%$ primary steel to be calculated:

$X_{p r}=\frac{X_{B O F^{-}}\left(\frac{\text { Scrap }_{B O F}}{\text { Scrap }_{r e}} X_{r e}\right)}{1-\frac{\text { Scrap }_{B O F}}{\text { Scrap }_{r e}}}$

This value for $X_{p r}$ can now be included in the scrap LCI equation and will therefore be applied to each of the inputs and outputs of the LCI. The values that have been used are based on the current worldsteel LCI data collection.

$$
X_{p r}=\frac{1.756-\left(\frac{0.119}{1.092} 0.386\right)}{1-\frac{0.119}{1.092}}
$$

It should be noted that if an extrapolation was carried out in order to determine the theoretical value for $X_{p r}$ with zero scrap input, based on the values of $X_{\mathrm{BOF}}$ and $X_{r e}$, the same values would be reached for $X_{p r}$ of $1.92 \mathrm{~kg} \mathrm{CO}_{2}$. Figure 6 plots the global EAF steel value which is based solely on steel scrap, together with the global BOF steel value which contains nearly $12 \%$ scrap. Extrapolating this to a value of zero scrap input gives this value of $1.92 \mathrm{~kg} \mathrm{CO}$.
Fig. 5 a Determination of the theoretical value of $100 \%$ primary BOF steel, $X_{p r}$ b Theoretical value of $100 \%$ primary BOF steel, $X_{p r}$ a

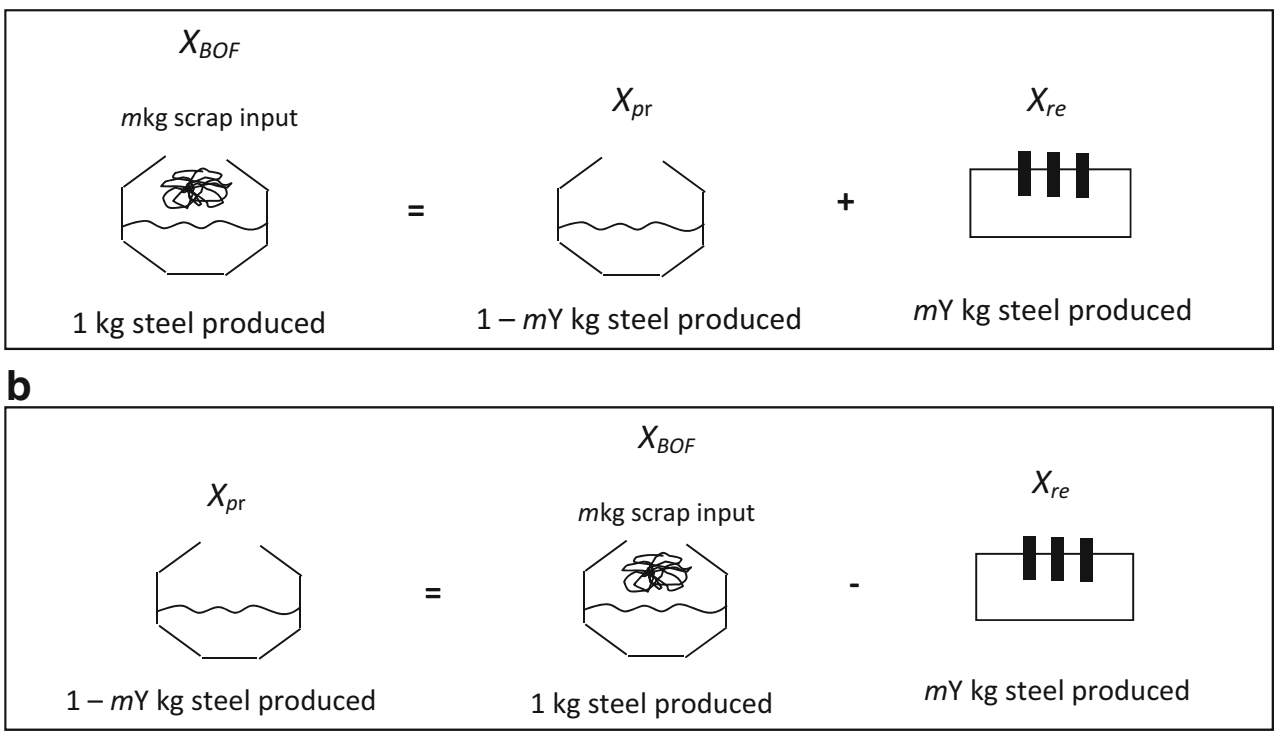


Fig. 6 Extrapolation to show $\mathrm{CO}_{2}$ emissions for $0 \%$ scrap input

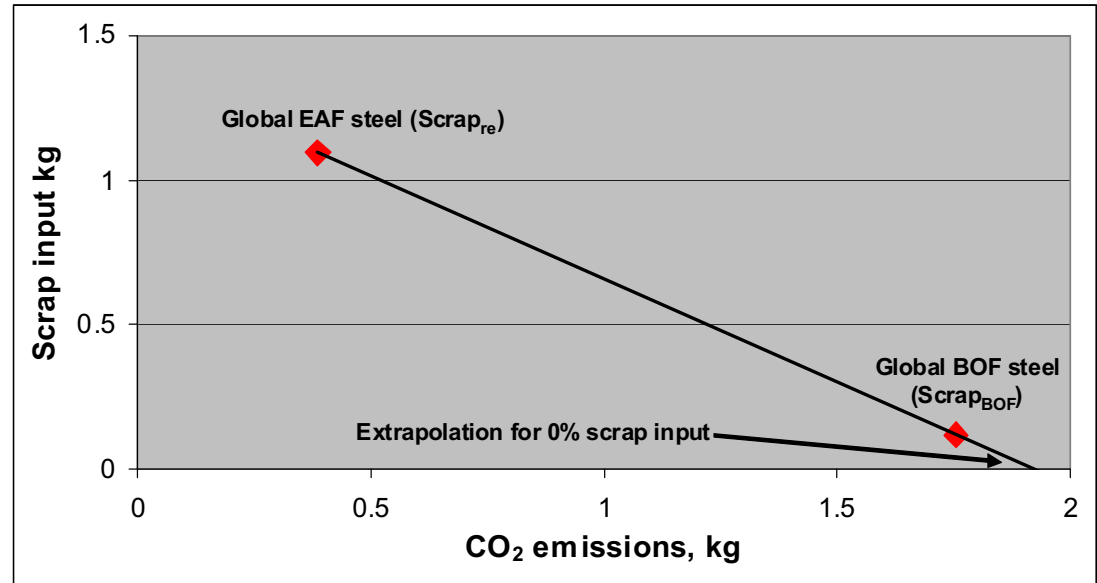

And for $\mathrm{CO}_{2}$, the equation would be as follows (i.e. $X=\mathrm{CO}_{2}$ ):

$$
\begin{gathered}
\text { ScrapLCI }=\left(X_{p r}-X_{r e}\right) Y \\
\text { ScrapLCI }=[1.92-0.386] \frac{1}{1.092} \\
\text { Scrap LCI }=1.405 \mathrm{kgCO}_{2}
\end{gathered}
$$

\subsection{Summary of scrap LCI calculations}

The methodology for determining the LCI for steel scrap, as described in Sects. 6.2 and 6.3, is summarised in Fig. 7. The figure uses $\mathrm{CO}_{2}$ as an example and includes the scrap inputs to the EAF and BOF processes to calculate the LCI for each production route when including a burden for the scrap. As the impact if the two routes can be equated when the burden for scrap has been included, this means that the scrap LCI can then be calculated.

\subsection{Applying the scrap LCI burden and credit}

The scrap LCI, defined in Eq. (1) as $\operatorname{Scrap} L C I=\left(X_{p r}-X_{r e}\right) Y$, is applied to the steel product cradle to gate LCIs in order to include the end-of-life phase. A credit is given for the amount of steel scrap that will be recycled at the end-of-life of the product, and this is referred to as RR. However, in doing this, a burden needs to be applied to any scrap that is used in the steelmaking process, referred to as $S$.

Thus, the LCI of a product, from cradle to gate including end-of-life ( $\left.\mathrm{LCI}_{\text {including EoL }}\right)$, can be calculated as

$$
L C I_{\text {includingE } o L}=X-(R R-S)\left(X_{p r}-X_{r e}\right) Y
$$

Fig. 7 Overview of scrap LCI calculations

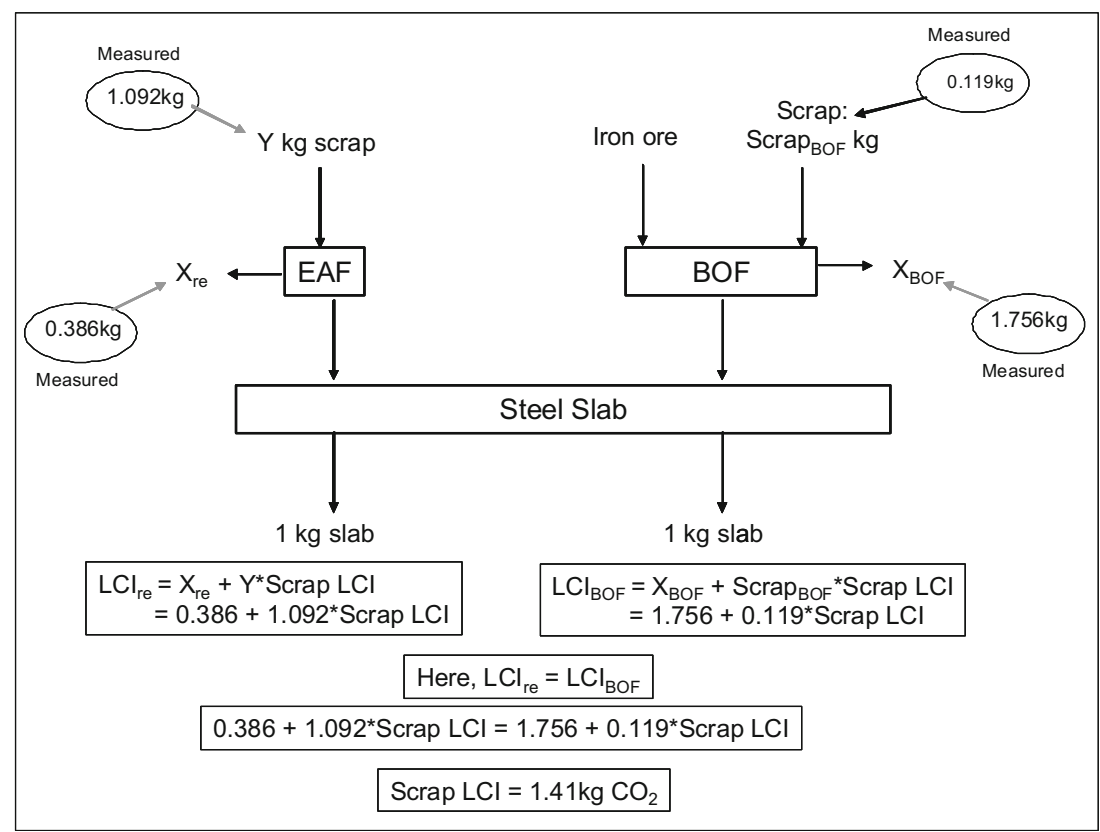




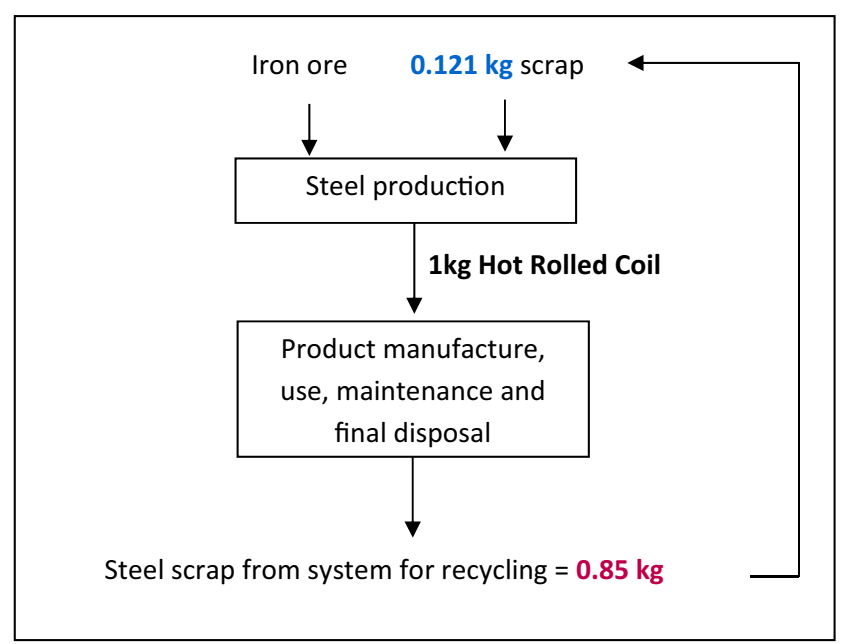

Fig. 8 Example cradle to grave system

where $X$ is the LCI of the product being studied and is cradle to gate, i.e. including all upstream as well as steel production. The term $(\mathrm{RR}-\mathrm{S})$ is also known as the net scrap that is generated from the product system. When this value is negative that implies that there is more scrap consumed to make the steel than is recycled from the product at the end-of-life.

In order to calculate the LCI of a steel product, including end-of-life recycling, an example for $\mathrm{CO}_{2}$ emissions is shown in Fig. 8 and Eq. (11), for global hot rolled coil, using an endof-life recycling rate of $85 \%$. This gives a net scrap value of $0.85-0.121=0.729 \mathrm{~kg}$.

The value of scrap, $\left(X_{p r}-X_{r e}\right) Y$, has been calculated above, and the $\mathrm{CO}_{2}$ emissions and scrap content of hot rolled coil are provided from the global average data published in February 2010. New data will be available at the end of 2015.

$$
\begin{gathered}
L C I_{\text {includingE } o L}=1.889-(0.85-0.121) * 1.405 \\
L C I_{\text {includingE oL }}=0.86 \mathrm{kgCO}_{2}
\end{gathered}
$$

$\mathrm{CO}_{2}$ is used in this example as it is one of the most commonly used LCI flows. The same calculation method applies to all inputs and outputs of the LCI.

\section{Conclusions}

The steel industry is an integral part of the circular economy model, and steel has fundamental advantages as a material in achieving this goal. The promotion of zero waste, reducing the amount of resources and energy used, making products that are easier to reuse or remanufacture, and finally being able to recycle steel from all parts of a products life make steel an essential material for the future. The methodology described here addresses the recycling aspect of the circular economy as well as the zero waste aspect. By demonstrating the benefits of recycling steel, it is evident that this practise should be encouraged and enabled through improved design of products, so that once they have been reused or remanufactured, the steel parts can easily enter into the recycling stream, reducing the need for primary raw materials.

However, due to the long life of steel products, the amount of steel in stock is a limiting factor in terms of what is available for recycling. Therefore, it is necessary to continue with primary steel production in order to meet the demands for steel. In addition, as more scrap is being used, attention must be paid to the proper sorting of the scrap to ensure that the higher quality steel grades can be achieved.

The steel industry is currently looking into product-related indicators, and this can incorporate indicators that address the circularity of steel in product applications. An indicator reflecting the benefits of recycling steel at the end of its life would show the contribution of using recyclable materials to achieve a circular economy.

There is scope for the steel industry to engage with its customers to improve the yield during the manufacturing processes as well as to design products which are easier to reuse, remanufacture and recycle. Scrap collection facilities should be improved to continue to increase the amount of scrap that is being recovered. Ongoing efforts to improve the environmental performance of steel production are also the key. This will all contribute to the goal of achieving a circular economy.

Open Access This article is distributed under the terms of the Creative Commons Attribution 4.0 International License (http:// creativecommons.org/licenses/by/4.0/), which permits unrestricted use, distribution, and reproduction in any medium, provided you give appropriate credit to the original author(s) and the source, provide a link to the Creative Commons license, and indicate if changes were made.

\section{References}

Atherton J et al (2007) Declaration by the metals industry on recycling principles. Int J Life Cycle Assess 12(1):59-60

BSI British Standards Institute (2008) PAS 2050"Specification for the measurement of the embodied greenhouse gas emissions of products and services" on Carbon footprinting. And: BSI British Standards (with DEFRA and Carbon Trust) (2008). Guide to PAS 2050 - How to assess the carbon footprint of goods and services. ISBN 978-0580-64636-2

Ellen MacArthur Foundation (2014) www.ellenmacarthurfoundation.org European Commission (2010) European platform on LCAhttp://eplca.jrc. ec.europa.eu/

European Commission (2013) Product environmental footprint, http://ec. europa.eu/environment/eussd/smgp/product_footprint.htm

European Committee for Standardisation (2013) CEN TC 350: sustainability of construction works

ISO TS 14067 (2013) Carbon footprint of products

The Greenhouse Gas Protocol (2004) World Resources Institute/World Business Council for Sustainable Development: Corporate Standard and Product Standard developed under the GHG Protocol Initiative

World Economic Forum (2014) Scoping paper: mining and metals in a sustainable world 